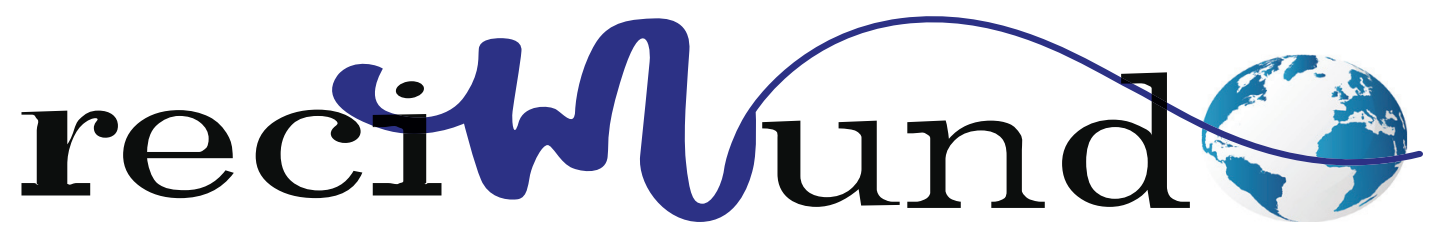

Revista Científica Mundo de la Investigación y el Conocimiento

DOI: 10.26820/recimundo/4.(4).octubre.2020.434-444

URL: http://recimundo.com/index.php/es/article/view/961

EDITORIAL: Saberes del Conocimiento

REVISTA: RECIMUNDO

ISSN: 2588-073X

TIPO DE INVESTIGACIÓN: Artículo de Revisión

CÓDIGO UNESCO: 5801 Teoría y Métodos Educativos

PAGINAS: 434-444

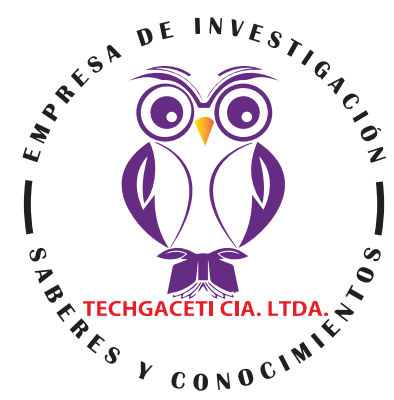

\title{
Enseñanza por competencia, desafío de la educación universitaria del siglo XXI
}

Teaching by proficiency, a challenge for university education in the 21 st century Ensinar por proficiência, um desafio para o ensino universitário no século XXI Delia Georgina Bravo Bonos ${ }^{01}$; Doris Susana Delgado Bernal2; Gioryi Augusto Sornoza Zavala3; Yadira Vanesa Yaguana Martínez ${ }^{4}$

RECIBIDO: 10/07/2020 ACEPTADO: 26/08/2020 PUBLICADO: 15/10/2020

1. Magíster en Urgencias Médicas; Licenciada en Enfermería, Universidad Etatal del Sur de Manabí, Jipijapa, Ecuador; delia. bravo@unesum.edu.ec; (D) https://orcid.org/0000-0003-4787-8403

2. Magíster en Gerencia en Salud; Licenciada en Enfermería; Universidad Estatal del Sur de Manabí; Jipijapa, Ecuador; doris. delgado@unesum.edu.ec; (D) https://orcid.org/0000-0001-5614-2567

3. Magíster en Psicología Laboral; Psicólogo Clínico; Universidad Estatal del Sur de Manabí; Jipijapa, Ecuador; giorgy.sornoza@unesum.edu.ec; (iD https://orcid.org/0000-0002-5573-3119

4. Maestrante en Docencia Universitaria; Especialista en Pediatría; Universidad Europea de Madrid; Madrid, España; yymar_141@hotmail.com; (iD https://orcid.org/0000-0003-3914-1540

\section{CORRESPONDENCIA \\ Delia Georgina Bravo Bonoso \\ delia.bravo@unesum.edu.ec}

Jipijapa, Ecuador

๑) RECIMUNDO; Editorial Saberes del Conocimiento, 2020 


\section{RESUMEN}

El vertiginoso desarrollo tecnológico ha impactado hacia modelos de producción más especializados por lo que, la competencia en el ámbito laboral exige de profesionales cada vez con mayores destrezas; la enseñanza por competencias en las universidades, constituye el desafío de nuestra época, convirtiéndose en la estrategia pedagógica que acierta la polémica de la formación vocacional y que responde a las necesidades del mundo cambiante del siglo XXI. Este modelo educativo desarrollado en el mundo entero, ha tenido principal auge en Europa, adoptado en virtud de la libre movilidad de los profesionales del territorio de la Unión Europea, por lo que los principales estudios refieren a la experiencia que aporta el viejo continente. En el marco de este modelo, gira la histórica polémica sobre la educación profezionalizante y la educación humanista. En torno a este debate, surge el presente estudio de investigación documental para identificar principales avenencias y desafíos de este modelo, proporcionando aportes teóricos que permita al profesional en materia, la profundización del estudio de la enseñanza por competencia, y aporte a la adecuación requerida en función de su efectiva aplicabilidad como respuesta a la educación que reclama el mundo de hoy.

Palabras clave: Competencias, Educación, Enseñanza, Pedagogía, Educación Superior.

\section{ABSTRACT}

The vertiginous technological development has impacted towards more specialized production models, therefore, competition in the workplace requires professionals with increasingly greater skills; teaching by competencies in universities constitutes the challenge of our time, becoming the pedagogical strategy that succeeds in the controversy of vocational training and that responds to the needs of the changing world of the 21st century. This educational model developed throughout the world, has had the main boom in Europe, adopted by virtue of the free mobility of professionals from the territory of the European Union, so the main studies refer to the experience provided by the old continent. Within the framework of this model, the historical controversy about professionalizing education and humanistic education revolves. Around this debate, the present study of documentary research arises to identify main avenues and challenges of this model, providing theoretical contributions that allow the professional in this matter, the deepening of the study of teaching by competence, and contribution to the adaptation required in function of its effective applicability as a response to the education demanded by the world today.

Keywords: Competences, Education, Teaching, Pedagogy, Higher Education.

\section{RESUMO}

O vertiginoso desenvolvimento tecnológico teve impacto em modelos de produção mais especializados, portanto, a competição no local de trabalho exige profissionais com competências cada vez maiores; o ensino por competências nas universidades constitui o desafio do nosso tempo, tornando-se a estratégia pedagógica que consegue a polémica da formação profissional e que responde às necessidades do mundo em mudança do século XXI. Este modelo educativo desenvolvido em todo o mundo, teve o principal boom na Europa, adoptado em virtude da livre mobilidade dos profissionais do território da União Europeia, pelo que os principais estudos se referem à experiência proporcionada pelo velho continente. No âmbito deste modelo, gira a controvérsia histórica sobre a profissionalização da educação e da educação humanista. Em torno deste debate, surge o presente estudo de investigação documental para identificar as principais vias e desafios deste modelo, fornecendo contributos teóricos que permitem ao profissional nesta matéria, o aprofundamento do estudo do ensino por competência, e o contributo para a adaptação necessária em função da sua efectiva aplicabilidade como resposta à educação exigida pelo mundo de hoje.

Palavras-chave: Competências, Educação, Ensino, Pedagogia, Ensino Superior. 


\section{Introducción}

En la última década la educación universitaria ha dado un giro, en la en búsqueda de métodos más efectivos, situando a la enseñanza por competencias, como la estrategia pedagógica que acierta en la polémica de la formación vocacional. Y es que, ésta procura identificar las capacidades y habilidades del estudiante y emprende el proceso cognitivo en función del desarrollo de las mismas, obteniendo resultados más idóneos ante las exigencias del mercado laboral y el desarrollo técnico-productivo actual. Motivo por lo que el siglo XXI ha significado la adecuación de los modelos educativos en virtud de la capacidad vocacional, de habilidades y de competencias.

Aún y cuando algunos autores plantean que la enseñanzas por competencia obedece más a un hecho meramente económico, es innegable la importancia que este método reviste, dada el creciente auge, y el interés por parte de sector productivo, gubernamental y educativo ya que el aporte de los profesionales ha mejorado su desempeño laboral ante los cambios tecnológicos acaecidos en el aparato industrial en la actualidad.

Ciertamente, se establecen marcadas diferencias al respecto, estas radican en que algunos autores plantean, según (Escudero, 2009), que los métidos de enseñanzas deben dar "un mayor reconocimiento del papel y el valor de la educación en la sociedad del conocimiento y busca reconstruir los sistemas educativos de forma radical, con el objetivo de garantizar a todas las personas una educación de mayor calidad, más justa y equitativa al mismo tiempo" .Mientras que la otra postura, tal señala (Escudero, 2009)

"Reconoce el valor estratégico de la educación en la economía del conocimiento, lo que se busca es la excelencia, el sometimiento de la formación a los imperativos de la globalización económica y las demandas del mercado, la innovación y la competitividad mercantil, la racionalización, eficiencia y eficacia de los sistema escolares en todos sus niveles, el aprendizaje a lo largo de toda la vida"

En un mundo globalizado y con cambios tecnológicos tan radicales, la competencia que se manifiesta en el ámbito de mercado laboral, requiere una mayor dedicación a este último planteamiento, preparando al estudiante, o en palabras de (López, Benedito, \& León, 2016)"Se requiere un sistema que reconozca la capacidad de desempeñarse efectivamente en el trabajo y no que sólo considere los conocimientos adquiridos."

Este modelo educativo ha tenido principal auge en Europa, en donde los paises lo adoptaron y es que el planteamiento de la educación por competencia se posicionó como estrategia académica de la Unión Europea para garantizar la libre movilidad profesional entres sus países miembros; se puede mencionar que tal disyuntiva se manifiesto, tal como aseveran el estudio realizado por (López, Benedito, \& León, 2016) quienes aseveran que "la formación universitaria en España ha estado marcada por la supremacía del modelo académico de formación frente al modelo que han denominado como profesionalizante".

Este hecho ha generado brechas entre el enfoque disciplinar y humanista de la formación universitaria y el enfoque por competencias, basado en la formación de profesionales. "El primero, más centrado en el saber, ha gozado de elevado prestigio y reconocimiento social, mientras que la formación profesional, más interesada en impulsar el saber hacer, no ha disfrutado del mismo status"(López, Benedito, \& León, 2016).

En efecto, esta distinción de lo académico separado de la formación hacia lo laboral 
conlleva en sí una histórica polémica de desencuentros y prejuicios, debate quemuchas veces desvirtúa que en efecto, la universidad profesionaliza, o educa en función de crear los profesionales que requiere la sociedad. Es decir, las habilidades, conocimientos y actitudes que la formación debiera proveer al individuo, deben ser aquellas en donde el mismo, se pueda desempeñar aportando al medio productivo que se traduce en pro de la sociedad. En todo caso, no debe desconocer los avances formativos del ser humano en la educación universitaria, bajo la visión de una formación academicahumanista y de calidad.

Así entonces, es menester el desarrollo de estudios como el presente, que pretende profundizar en este enfoque educativo desde una perspectiva menos antagonica y en función de servir como aporte teorico a la fomaciónuniversitaria. Se pasea por algunas viscicitudes que se ha encontrado este tipo de enseñanza como lo son : "a falta de formación docente del profesorado universitario que dificulta el compromiso del colectivo docente con el cambio, sino que sitúa al profesorado universitario en una posición de debilidad desde la que resulta complejo hacer frente a los desafíos y dilemas que conlleva el enfoque de competencias y su traslación a la práctica educativa" (López, Benedito, \& León, 2016).

Por otra parte, se estudia la evaluación del enfoque de competencias, pues la calidad del aprendizaje y el nivel de logro de cualquier competencia depende entre otros aspectos, de la pedagogía y de los procesos de evaluación. De tal forma, que el propósito de la presente investigación consiste en analizar la información documental sobre el enfoque de competencias adoptado en la educación universitaria y, su contribución a la calidad educativa.

\section{Metodología}

El presente analisis se plantea la investiga- ción documental , mediante revisión bibliográfica y documental, en medios electronicos, artículos científicos y demás fuentes hemerofraficas aue permirten ahondar en el modelo de enseñanza por competencias en la educación universitaria, indagando sobre sus procesos de evaluación y su aplicabilidad.

Se analiza estudio de tipo descriptivo realizado por (López, Benedito y León, 2016) en la Universidad Autónoma de Barcelona, de Cádiz, de Barcelona, de Deusto, de Granada, de Murcia y Universidad de Valladolid; donde en base a la experiencia se consultó a expertos titulares que han desarrollado investigaciones y publicaciones en los últimos años sobre el enfoque de competencias en la educación universitaria, en virtud de identificar viscitudes y aportes del sistema de evaluación de competencias basado en su aplicabilidad y la experiencia.

\section{La Competencia}

La noción de competencias han desarrollado distintas definiciones la competencia hace referencia a la ejecución de tareas, la pericia y habialidades en la realización de determinadas tareas, algunas de las definiciones, dada por distintos autores se pueden destacar:

- "Un saber actuar complejo que se apoya en la movilización y la combinación eficaz de una variedad de recursos internos y externos dentro de una familia de situaciones" (Tardif, 2006)

- "Una competencia tiene como característica ser integradora, combinatoria, en desarrollo, contextual y evolutiva". (Fernández March, 2011)

La competencia vincula diversos recursos de naturaleza variada, de allí su carácter integrador que evidencia la complejidad del saber actuar, la cual implica movilización y la combinación eficaz de determinados recursos que se complementen y hagan

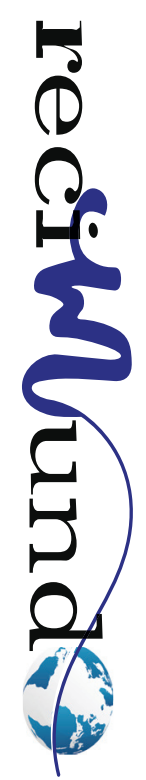


sinérgia, para su fijación y aprendizaje. En efecto, se pues aseverar que:

- La competencia evoluciona con el transcurso del tiempo, De tal forma que debe tenerse en cuenta el nivel de desarrollo que debe alcanzarse para cada una de las competencias al finalizar la formación universitaria, entendiendo que por su carácter sistémico y evolutivo, los profesonales desarrollarán habilidades en torno a la misma, también en la vida profesional.

- En tal sentido, el nivel alcanzado, del desarrollo de la habilidades y competencias, será lo que oriente la elección de las situaciones de aprendizaje, métodos y las modalidades a ejercer de evaluación.

El carácter contextual de la competencia da un sentido al aprendizaje que se vuelve consciente y reflexivo. Las situaciones imponen una elección en la movilización y la combinación de los recursos. Son el detonador y el marco de acción. Los docentes tienen entonces la responsabilidad de elegir juiciosamente las situaciones a las que quieren exponer a sus estudiantes. Finalmente, una competencia cuenta también con un carácter evolutivo ya que a medida que la persona acumula recursos, la movilización y la combinación de los mismos evoluciona con el tiempo para asegurar su eficacia en situaciones cada vez más complejas.

Al respecto, señala (Catalano, Avolio de Cols, \& Sladogna, 2004, pág. 39) que la enseñanza por competencia es concebida como "la capacidad de movilizar conocimientos y técnicas y de reflexionar sobre la acción. Es también la capacidad de construir esquemas referenciales de acción o modelos de actuación que faciliten las acciones de diagnóstico o de resolución de problemas productivos no previstos o no prescritos".
En efecto, la competencia refiere a un sistema de conocimientos, habilidades y actitudes que deben ser utilizados de modo interactivo en la ejecución oportuna de tareas y en la resolución de problemas, en una situación dada, lo que implica movilizar recursos y medios disponibles en el ambiente específico en que se opera. Esto puede desempeñarse en lo académico tanto como en laboral. En tal sentido, el sujeto no usa una única competencia sino que a causa de su naturaleza global y en función del nivel de complejidad de la tarea ejecutada o problema abordado se suelen utilizar de modo conjunto varias competencias en paralelo (López Ruiz, 2011).

Las competencias puestas en desarrollo conllevaran a resultados postivos o no, sobre los objetivos ha alcanzar, de tal manera, que el desarrollo cognitivo de la habilidad, su entrenamiento, practica y aprehensión de criterios a emplear o que proporcionen la capacidad de respuesta, serán fundamental al alcance de ciertos logros positivos. Si los resultados o productos derivados del despliegue de las competencias no se consideran satisfactorios, se concluye que esa persona todavía no es competente.

Plantea (López Ruiz, 2011) que la competencias comprenden los siguientes elementos:

- Esquemas de acción: son modelos mentales de representación de la acción o de anticipación de resultados que el sujeto construye en función de los recursos personales y ambientales con los que cuenta

- Saberes: son los conocimientos que se precisan activar y aplicar en la situación problemática. Funcionan a su vez como un sistema de ideas o principios que se ponen en relación para interpretar y actuar en ese contexto.

- Saber hacer: son el conjunto de destrezas y habilidades que es necesario desplegar en ese momento o transferir a 
otros contextos y situaciones.

- Saber ser: se refieren al elenco de actitudes, valores, atributos personales y disposiciones que median la puesta en juego de los conocimientos y destrezas.

- Resultados positivos o favorables: es el estado final alcanzado después del desempeño eficaz de la tarea

En sinstesis, se puede precisar que las competencias se caracterizan por ser:

- Sistemáticas, ya que se relacionan a pesar de interactuar independientemente una de otra.

- Secuenciales: su evolución es progresiva, se debe ejercitar y entrenar su capacidad cognitiva y fijación para su cabal evolución y aumento de capacidad, o como lo señalará (López Ruiz, 2011) "por lo que siempre pueden ser perfeccionables".

- Funcional, ya que persigue como objetivo último el desarrollo de determinada función.

- Contextualizada, ya que se desarrolla de forma especifica según determinada situación y se puede transpolar a otro escenario que lo amerite, una vez fijada y dominada.

- Autentica.

- Proyección social, ya que busca aportar a la sociedad en su crecimiento y desarrollo.

- Innovadora, una vez con dominio cabal, se ejerce en consecuencia el proceso creativo de cambios.

Por su parte, cabe destacar las competencias en lo laboral tienen un enfoque desde la estructura empresarial y de la educación para el trabajo. Cada vez más se evidencian nuevas configuraciones en la forma de producir o generar los servicios y en el perfil de los trabajadores se exige mayores destrezas y desempeño técnico en le quehacer de la actividad productivas. Entre algunas de las categorías generales de competencias o competencias transversales que se manifiestan en el ambito lanoral, destacan: gestión de recursos, relaciones interpersonales, gestión de información, comprensión sistémica y dominio tecnológico. (Larraín U \& González F, 2007). Por su parte, (Marelli, 2000, pág. 7), ha definido la competencia como «capacidad laboral, medible, necesaria para realizar un trabajo eficazmente. Está conformada por conocimientos, habilidades, destrezas y comportamientos que los trabajadores deben demostrar para que la organización alcance sus metas y objetivos»

\section{Enseñanza por competencias:}

El vertiginoso avance tecnológico ha conllevado a la modernización de los procesos productivos, el uso de las TICs, las redes , la globalización y la internacionalización, han convertido a la formación curricular basada en competencias en el soporte fundamental de los cambios y transformaciones que se vienen produciendo en la Educación Superior a nivel mundial.

Es importante acotar lo definido por Tardif (2006) sobre el modelo cognitivo, el cual es "una modelización basada en datos científicos válidos, que, a partir de aprendizajes críticos, circunscribe las etapas del desarrollo de una competencia" (p. 55).

Así entones, la enseñanza por competencias ha significado la técnica para el desarrollo cognitivo a nivel profesional y ha sido utilizada para incrementar el desempeño laboral, en el ámbito empresarial, así como en la captación y educación de los empleados. Mientras que, en lo que respecta a la educación universitaria Rué (2007), señala que la implementación de este enfoque en este ámbito implica unos parámetros singulares que lo distinguen de la aplicación que hasta ahora ha tenido en los dos planos señalados. La noción de competencias no puede considerarse estrictamente unívoca por lo que se han desarrollado distintas definiciones, sobre todo hasta el momento, dentro

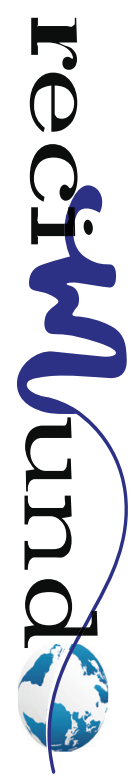


del área empresarial de recursos humanos y en el nivel de la Formación Profesional. Desde ambas perspectivas se pone el punto de mira en las capacidades y habilidades que una persona requiere para desempeñar un empleo concreto con eficiencia. Esto hace que sea necesario elaborar una conceptualización más específica en el campo de la Educación Superior, para integrar además de la pericia en la realización de las tareas profesionales los saberes fundamentales que se deben poseer desde una perspectiva académica. Por tanto, se trata de conseguir un delicado equilibrio entre la mejora del nivel de empleabilidad de los egresados y su elevada formación científica y humanística. Por consiguiente, el punto crucial de la enseñanza por competencia reside en el este punto crucial, lo cual la distingue la inclusión de las competencias en la Educación Superior del uso que ha tenido en los otros dos casos.

\section{Evaluación de las enseñanzas por cope- tencias}

El objeto de la evaluación no son sólo los conocimientos adquiridos sino también, las competencias desarrolladas por los estudiantes. Para ayudarnos a hacer esta transición, Tardif (2006) propone ver la evaluación de competencias como un planteamiento videográfico y no fotográfico. En efecto, no se trata tanto de emitir un juicio al final del trayecto como de seguir la progresión del mismo. En este sentido, la evaluación formativa, que informa al estudiante sobre la progresión de su aprendizaje, es un elemento esencial en la evaluación de las emseñanzas por competencias (Scallon, 2000).

La evaluación de competencias se basa entonces en el acceso a fuentes múltiples y variadas de información con el fin de determinar si los estudiantes han alcanzado el nivel esperado de desarrollo de competencias, así como un grado suficiente de dominio de los recursos vinculados a cada compe- tencia. Como la competencia es un "saber actuar complejo en situación", la evaluación de las competencias deberá realizarse en una situación lo más auténtica posible (Lussier y Allaire, 2004). Por situación auténtica, nos referimos a una situación lo más cercana posible al contexto profesional en el que los estudiantes podrán evolucionar una vez que hayan finalizado sus estudios. Estas situaciones auténticas de evaluación permiten a los estudiantes comprometerse en la realización de una tarea completa, compleja y significativa.

Si los resultados o productos derivados del despliegue de las competencias no se consideran satisfactorios, se concluye que esa persona todavía no es competente, diestra o experta en larealización de esa tarea o en la resolución de ese problema.

En estudio de tipo investigación narrativa, realizado por López, Benedito y León (2016) centrado en el análisis de las respuestas proporcionadas por expertos de universidades españolas, específicamente de la Universidad Autónoma de Barcelona, de Cádiz, de Barcelona, de Deusto, de Granada, de Murcia y Universidad de Valladolid; en donde se consulto a catedráticos y Titulares, con investigaciones y publicaciones en los últimos años sobre el enfoque de competencias en la educación universitaria sobre el sistema de evaluación de competencias basado en su experiencia, mencionaron de forma concluyente que los exámenes teóricos no son útiles para evaluar competencias. En cambio, señalan, como adecuados, los siguientes: portafolios, ensayos e informes, estudios de caso, resolución problemas, escalas, parrillas, rúbricas, debates, pruebas reflexivas, listas de control, exposiciones orales; ,instrumentos, muchos de ellos, ya señalados, como pertinentes, por otros autores (Brown y Glasner, 2010; Marín et al., 2013).

La evaluación de competencias se considera un factor clave de mejora de la formación 
si ésta no sólo se utiliza para calificar:

"La realización práctica de la evaluación ayuda a comprender la complejidad de la propia formación y las incoherencias que debemos evitar" (López, Benedito y León, 2016); la evaluación se convierte así en instrumento de formación y de práctica docente reflexiva. Los participantes en el estudio coinciden, por tanto, en considerar al profesorado un factor clave del cambio, pero reconocen que existe poca reflexión, debate y valoración sobre lo que realmente supone la adopción e implementación del enfoque por competencias profesionales. Esta falta de reflexión obstaculiza, en su opinión, el enfoque competencial y su correcta articulación en la práctica formativa y de evaluación, corriendo el riesgo de que este quede en un mero ejercicio intelectual baladí como advierten Marín et al. (2013). Otros factores a considerar son la necesidad de formación del profesorado y de coordinación académica. El experto 6 insiste en la "necesidad de la coordinación de equipos docentes en la discusión y reflexión sobre el proceso y las finalidades de la formación universitaria. Puesto que la evaluación es CLAVE y condiciona todo el proceso de e/a (enseñanza-aprendizaje)" Finalmente, los participantes del estudio consideran necesario diversificar los instrumentos de evaluación y opinan que la pertinencia del instrumento no viene determinada por el instrumento en sí, sino por el tipo de tarea a evaluar y la finalidad que se persigue.

De acuerdo con estos resultados, señalan (López, Benedito y León, 2016), que el personal experto del estudio reconoce que el enfoque competencial representa una oportunidad para superar el planteamiento excesivamente academicista de la enseñanza universitaria, ensalzar el valor formativo de la práctica y reforzar la conexión con la realidad profesional, virtualidades también destacadas por Tejada (2006) y Taylor y Hooley (2014). Asimismo, consideran que la lógica disciplinar y la fragmentación por materias representan un serio revés para el desarrollo de competencias, pues su naturaleza integral requiere propuestas formativas interdisciplinares, fruto del trabajo en equipo del profesorado y del apoyo de la institución universitaria, como sugieren Rué y Lodeiro (2010) y Agudo et al. (2013).

Aunado, indican como positivo, el protagonismo conferido al alumnado en el proceso de aprendizaje y la renovación que, a nivel didáctico, comporta para la enseñanza universitaria.

\section{Enseñanza por competencias en la edu- cación universitaria del siglo $\mathrm{XXI}$}

La disyuntiva sobre el valor de la educación, el "retorno de la inversión", señalado por (Facer, 2011, p. 25;UNESCO 2015), así como las tasas de deserción de estudiantes reflejan una incapacidad por parte de los sistemas educativos para vincular el contenido académico con el mundo real experiencia. (Scott, 2015)

De tal forma, que el modelo de la enseñanza por competencia debe basado en la experiencia de los profesores y de los profesionales en ejercicio, desarrollar ciertos aspectos, en virtud de consolidarse como la alternativa que exige la sociedad actual. Asi se pueden ennumerar:

1. Desarrollar cada una de las competencias contempladas en un programa,

2. Identificar los niveles de desarrollo de la enseñamza por competencia y aquello que caracteriza su fijación y perfeccionamiento.

3. Determinar cuales son los aprendizajes esenciales en cada uno de los niveles desde el nivel inicial hasta el nivel de experto.

La realización de esta fase precisa partir del enunciado de la competencia y de la descomposición de la misma en sus diferentes componentes que pueden ser de tipo conceptual, procedimental de primer nivel,

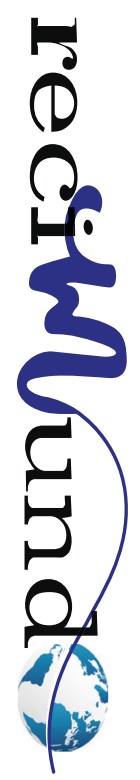


procedimental de segundo nivel (condicional) y actitudinal. Esta descomposición nos acerca más al concepto de resultado de aprendizaje.

En torno al desarrollo pedagógico en la educación universitaria del del siglo XXI ya apuntaba la UNESCO, en estudio desarrollado por (Scott, 2015) que debe contener, elementos como:

1. Integración multicultural para abordar la inmigración y cambio demográfico;

2. Reducir el abandono escolar prematuro para combatir el desempleo y promover una fuerza laboral mejor educada;

3. Fomentar el talento para desarrollar una economía "inteligente" basada en conocimiento e innovación;

4. Promover una transición rápida y fluida de la escuela trabajar para reducir las barreras entre el mundo del trabajo y la educación;

5. Facilitar el reingreso al mercado laboral especialmente para abordar el desempleo de larga duración;

6. Centrarse en la reconversión permanente para que todos los ciudadanos puedan mantener sus competencias actualizadas y responder rápidamente a los entornos laborales cambiantes (Scott, 2015, pág. 12)

Como se menciona, el siglo XXI requiere de modelos de enseñanza por comperencias que preparen al individuo para las complejidades y lo vertogimoso de los cambios. Sawyer (2006) enfatiza la importancia de preparar a los jóvenes para estos futuros variados y colaborar a través de culturas para abordar posibles problemas ambientales, sociológicos y dificultades políticas. Aprender a "comprender, adaptarse y prosperar en estos tiempos turbulentos se ha convertido una competencia crítica "(Carneiro, 2007, p. 151). El mundo es cada vez más interconectados y complejos, y requerirá respuestas multifacéticas. La respuesta de la educación a estos Los desafíos juga- rán un papel singular y activo en la configuración de la futuro.

\section{Conclusiones}

Las enseñanza por competencia en la actualidad representa la oportunidad de impulsar la innovación y el desarrollo tecnológico de las naciones para su desrrollo y crecimiento; el desconocimiento y la falta de formación de los profesores para desarrollar este método bajo la lógica requerida puede conllevar a egresar profesionales o alumnos que no estén instruidos con las habilidades y conocimientos para llevar una vida laboral satisfactoria y productiva. En consecuencia, las naciones están perdiendo oportunidades para preparar a los jóvenes para la ciudadanía y la economía está sufriendo una falta de innovación.

Las potencialidades y oportunidades que trae consigo el siglo XXI y todos los cambios tecnológicos, desarrollo de medios y herramientas, constituyen la posibilidad de reafirmar y sustanciar el rol de la educación en su capacidad para preparar y dotar a los jóvenes para estar en capacidad de abordar la complejidad social, económica y cuestiones ambientales. Así llevarle al desarrollo cienifico y al aprovechamiento de sus propias capacidades en este proceso, que le permite interactuar de forma apropiada con el entorno. Los desafíos del mundo real son muy complejos, a menudo mal definido e interdisciplinario por naturaleza, que abarca múltiples dominios (social, económico, político, ambiental, legal y ético). Los alumnos deben tener la oportunidad de reflexionar sobre sus ideas, perfeccionar sus habilidades analíticas, fortalecer su crítica y capacidad de pensamiento creativo y demostrar iniciativa. En particular, la capacidad de evaluar nuevos aportes y perspectivas, crear nuevas capacidades y fortalecer la autonomía será crucial.

La transformación de la educación en los nuevos modelos que exige la sociedad de 
hoy en día, requiere del propio aprendizaje del maestro y profesionales del area de la educación, es lo que proporcionará las técnicas y fomentara su aplicación en torno a las mallas curriculares, y a los procesos de evaluación, de tal forma de poder aplicarlo en los alumnos, una variedad de competencias y habilidades necesarias para tener éxito en la economía global moderna.

Por otro, la integración de las competencias en el proceso de enseñanza-aprendizaje en la Educación Superior representa una importante reforma que pretende el incremento de la calidad tanto de la docencia como de la gestión. De igual forma, en términos de pedagogía como de desarrollo docente, la inversión que produce innovaciones de aprendizaje exitosas en una nación puede tener un efecto dominó cuando otras naciones adoptan y adaptan estos métodos para su propio uso, constituyendose en una red de aprendizaje global acorde a las necesidades del mundo actual.

El método de la enseñanza por competencia, puede convertirse en el potencial aliado para el crecimiento económico de las naciones, por que desarrolla las destrezas en base a los requerimientos productivo d las naciones. De ta forma, que no debe escatimarse en investigaciones y aportes teóricos que apunte a las técnicas y pedagogía de la misma, en virtud de dar soluciones a las vicisitudes con las que se ha encontrado, en los países que vienen aplicándola durante las últimas décadas.

\section{Bibliografía}

Álvarez Junco, D. S., Peña Estrada, D. C., \& Palma Cardoso, D. E. (2020). Didáctica digital docente: mismo paradigma educativo, diferente medio. revista científico - educacional de la provncia Granma, 16.

Catalano, A. M., Avolio de Cols, S., \& Sladogna, M. (2004). Diseño curricular basado en normas de competencia laboral: conceptos y orientaciones. Banco Interamericano de Desarrollo.

de la Torre, S., \& Violant, V. (s.f.). ESTRATEGIAS
CREATIVAS EN LA ENSEÑANZA UNIVERSITARIA. Universidad de Barcelona.

Escudero, J. M. (2009). Las Competencias Profesionales y la Formación Universitaria. Revista Interuniversitaria de Pedagogía Social(16), 65-82.

Fernández March, A. (2011). LA EVALUACIÓN ORIENTADA AL APRENDIZAJE EN UN MODELO DE FORMACIÓN POR COMPETENCIAS EN LA EDUCACIÓN UNIVERSITARIA. Revista de Docencia Universitaria, 8(1).

Larraín U, A. M., \& González F, L. E. (2007). FORMACION UNIVERSITARIA POR COMPETENCIAS.

Le Boterf, G. (2002). Développer la compétence des professionnel. Éditions d'Organisation.

López Ruiz, J. I. (2011). Un giro copernicano en la enseñanza universitaria: formación por competencias. Revista de Educación, 279-301.

López, C., Benedito, V., \& León, M. J. (2016). El Enfoque de Competencias en la Formación Universitaria y su Impacto en la Evaluación. La Perspectiva de un Grupo de Profesionales Expertos en Pedagogía. Formación Universitaria, 9(4), 11-22.

Marelli, A. F. (2000). Introducción al análisis y desarrollo de modelos de competencias.

Osandón, L., Caro, M., Magendzo, A., Abraham, M., Lavín, S., González, F., \& Cabaluz, J. F. (julio de 2018). Estado, mercado y currículum escolar: la experiencia chilena (1964-2018). Cuestiones fundamentales y actuales del currículo, el aprendizaje y la evaluación(20). Oficina Internacional de Educación de la UNESCO.

Paredes Chacín, ,. A., Inciarte González, A., \& Walles Peñaloza, D. (Julio-Septiembre de 2020). Educación superior e investigación en Latinoamérica: Transición al uso de tecnologías digitales por Covid-19. Revista de Ciencias Sociales (RCS). FCES - LUZ, XXVI(3), 98-117.

Propuesta de la UNESCO para garantizar la educación online durante la pandemia. (2020). Obtenido de Educa web.

Scott, C. L. (2015). WHY MUST LEARNING CONTENT AND METHODS CHANGE IN THE 21st CENTURY? EDUCATION RESEARCH AND FORESIGHT.

Tardif, J. (2006). UN pasaje obligé dans la planification de l'évaluation des compétences: déterminer des indicateurs progressifs et terminaux de développement ( $2^{\mathrm{a}}$ partie). Pédagodie collégiale, 18(2), 13-20.

UNESCO. (2 de Septiembre de 2013). Universalidad de Internet: un medio para crear sociedades del

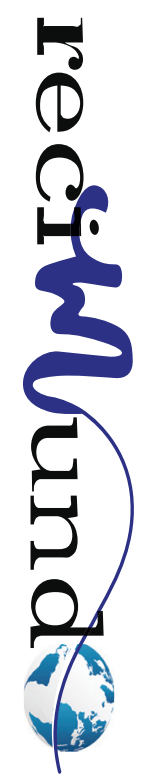


conocimiento y la agenda de desarrollo sostenible después de 2015.

United Nations. (2020). Policy Brief: Education during COVID-19 and beyond. United Nations.

\section{CITAR ESTE ARTICULO:}

Bravo Bonoso, D. G., Delgado Bernal, D. S., Sornoza Zavala, G. A., \& Yaguana Martínez, Y. V. (2020). Enseñanza por competencia, desafío de la educación universitaria del siglo XXI. RECIMUNDO, 4(4), 434-444. https://doi. org/10.26820/recimundo/4.(4).octubre.2020.434-444

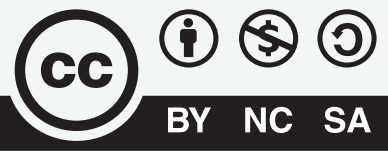

CREATIVE COMMONS RECONOCIMIENTO-NOCOMERCIAL-COMPARTIRIGUAL 4.0 . 